\title{
Accuracies of breeding values for dry matter intake using nongenotyped animals and predictor traits in different lactations
}

\author{
C. I. V. Manzanilla-Pech, ${ }^{*} \dagger^{1}$ R. F. Veerkamp, ${ }^{*}$ Y. de Haas, ${ }^{*}$ M. P. L. Calus,${ }^{*}$ and J. ten Napel ${ }^{*}$ \\ *Animal Breeding and Genomics, Wageningen University and Research, PO Box 338, $6700 \mathrm{AH}$ Wageningen, the Netherlands \\ †Mococha Research Station, National Institute of Forestry, Agriculture and Livestock Research, 97454 Mococha, Yucatan, Mexico
}

\begin{abstract}
Given the interest of including dry matter intake (DMI) in the breeding goal, accurate estimated breeding values (EBV) for DMI are needed, preferably for separate lactations. Due to the limited amount of records available on DMI, 2 main approaches have been suggested to compute those EBV: (1) the inclusion of predictor traits, such as fat- and protein-corrected milk (FPCM) and live weight (LW), and (2) the addition of genomic information of animals using what is called genomic prediction. Recently, several methodologies to estimate EBV utilizing genomic information (EBV) have become available. In this study, a new method known as single-step ridge-regression BLUP (SSRR-BLUP) is suggested. The SSRR-BLUP method does not have an imposed limit on the number of genotyped animals, as the commonly used methods do. The objective of this study was to estimate genetic parameters using a relatively large data set with DMI records, as well as compare the accuracies of the EBV for DMI. These accuracies were obtained using 4 different methods: BLUP (using pedigree for all animals with phenotypes), genomic BLUP (GBLUP; only for genotyped animals), single-step GBLUP (SS-GBLUP), and SSRR-BLUP (for genotyped and nongenotyped animals). Records from different lactations, with or without predictor traits (FPCM and LW), were used in the model. Accuracies of EBV for DMI (defined as the correlation between the EBV and pre-adjusted DMI phenotypes divided by the average accuracy of those phenotypes) ranged between 0.21 and 0.38 across methods and scenarios. Accuracies of EBV for DMI using BLUP were the lowest accuracies obtained across methods. Meanwhile, accuracies of EBV for DMI were similar in SS-GBLUP and SSRR-BLUP, and lower for the GBLUP method. Hence, SSRR-BLUP could be
\end{abstract}

Received February 16, 2017.

Accepted July 16, 2017.

${ }^{1}$ Corresponding author: cmanzanillap@gmail.com used when the number of genotyped animals is large, avoiding the construction of the inverse genomic relationship matrix. Adding information on DMI from different lactations in the reference population gave higher accuracies in comparison when only lactation 1 was included. Finally, no benefit was obtained by adding information on predictor traits to the reference population when DMI was already included. However, in the absence of DMI records, having records on FPCM and LW from different lactations is a good way to obtain EBV with a relatively good accuracy.

Key words: genomic prediction, feed intake, fat- and protein-corrected milk, live weight

\section{INTRODUCTION}

Feed costs represent half of the total costs of dairy production (European Union, 2011). One way to increase profitability of dairy production is to reduce feed costs by improving feed efficiency (Veerkamp, 1998; de Haas et al., 2012). Optimization of dairy cattle breeding goals for feed efficiency requires the availability of breeding values for DMI, as this is an important component of feed efficiency. To estimate accurate DMI breeding values, a large number of records is required. However, DMI is labor intensive and expensive to measure. As DMI is not a trait typically recorded in commercial herds, the amount of available data is limited. The difficulty in recording DMI has hampered direct selection for DMI previously because insufficient records were available on daughters of progeny-tested bulls. This difficulty might be overcome by jointly using predictor traits (Veerkamp and Brotherstone, 1997; Berry and Crowley, 2013; Manzanilla-Pech et al., 2016) and genomic information (Meuwissen et al., 2001). Readily available predictor traits that are easier and cheaper to record compared with DMI are fat- and protein-corrected milk (FPCM) and live weight (LW). Both traits are known to be strongly correlated with DMI (Korver, 1988; Van Arendonk, 1991; Veerkamp and Brotherstone, 1997; Veerkamp, 1998; Liinamo et al., 2012). 
Ideally, breeding values for DMI across the whole productive lifetime of cows should be predicted, but historical DMI data in the Netherlands have mainly covered just first lactations. It has been reported that DMI varies not only during lactation but also across lactations (Berry et al., 2006). For this reason, it is important to investigate the effect on the EBV of either including data (on DMI, FPCM, and LW) from only lactation 1, or using data from 3 lactations when predicting DMI in lactation 1 or in the first 3 lactations.

Best linear unbiased prediction is a widely used method in genetic improvement of livestock that uses pedigree relationships to estimate the EBV of an animal. In our study, we used this method as the base traditional method to compare the other BLUP methods [genomic BLUP (GBLUP), single-step GBLUP (SS-GBLUP), and single-step ridge-regression BLUP (SSRR-BLUP)].

Genomic BLUP uses genomic relationships to estimate the genetic merit of an individual (VanRaden, 2008). The genomic relationship matrix (GRM) constructs the relationship between individuals based on genomic information, instead of pedigree information. The main limiting factor of this method is that only phenotypic information of genotyped animals is used, thereby ignoring important phenotypic information from nongenotyped relatives.

Single-step GBLUP (Aguilar et al., 2010; Christensen and Lund, 2010) allows for adding phenotypic information of nongenotyped animals into the GBLUP model. This method combines, in a single step, the GRM $(\mathbf{G})$ with the pedigree relationship matrix $(\mathbf{A})$ into a new relationship matrix $(\mathbf{H})$, whose inverse is used to solve the mixed model equations to obtain the EBV. The SS-GBLUP is a well-known method due to its simplicity, which has been adopted in several countries for routine evaluations. However, this method has the limiting factor (in common with ordinary GBLUP) that the computation time required to invert the G-matrix more than quadratically increases with the number of genotyped animals.

In this work, a novel method will be evaluated being implemented in the software package MiXBLUP, which combines information about genotyped and nongenotyped animals with phenotypes using SNP estimates, instead of GRM, using BLUP methodology, namely, SSRR-BLUP. This methodology is based on the theory behind a previously presented Bayesian regression method (Fernando et al., 2014). The Bayesian regression method uses imputed SNP genotypes for animals that are not genotyped, together with a residual genetic effect of the deviations between true and imputed genotypes (Fernando et al., 2014). In theory, the accuracy of prediction of SSRR-BLUP is expected to be identical to SS-GBLUP, but its advantage is that it does not require the computation of $\mathrm{G}$ or its inverse.

In 2016, a breeding value for DMI was introduced in the Netherlands. As a consequence, more historical DMI data have been collected and combined for genetic analysis. Therefore, the objective of this study was to estimate genetic parameters using this uniquely large data set with DMI records, as well as compare accuracies in the prediction of the EBV of DMI, obtained by applying 4 different methods (i.e., BLUP, GBLUP, SSGBLUP, and SSRR-BLUP) using records from different lactations, with or without predictor traits (FPCM and LW), in the model.

\section{MATERIALS AND METHODS}

\section{Phenotypes}

Data Collection. A total of 459,096 daily records on DMI were available from 3,954 Dutch dairy cows in 6,820 lactations. These records came from cows that participated in nutritional experiments, which were conducted from 1987 to 2015 on several farms in the Netherlands (e.g., Aver Heino, Heino; Bosma Zathe, Ureterp; Cranendonck, Soerendonk; 't Gen, Lelystad; Minderhoudhoeve, Swifterbant; Waiboerhoeve Dairy Unit 2, 3, Lelystad; Zegveld farm, Zegveld; Hoorn, Lelystad; and New Wairboerhoeve, Lelystad). A full description of the methodology of most of the experiments and diets has been summarized previously (Veerkamp et al., 2000; Beerda et al., 2007; Zom et al., 2012). Diets primarily included grass silage, fresh grass, dehydrated grass, corn, corn silage, cereal, concentrates, or beet pulp. All cows were kept indoors in conventional cubicle housings, offered complete mixed rations ad libitum and milked twice a day, except for $50 \%$ of the cows at Bosma Zathe $(\mathrm{n}=50)$, which were milked 3 times per day.

Recorded Traits. Recording frequencies of DMI varied by experiment: it was recorded 1, 2, 3, or 5 times per week (Veerkamp et al., 2000; Beerda et al., 2007; Zom et al., 2012). An overview of the experiments, treatments and diets is summarized in Manzanilla-Pech et al. (2014). Weighing platforms were used to automatically monitor LW 3 times a week or daily, depending on the experiment. Fat- and protein-corrected milk was calculated, using milk yield and fat and protein content. Milk yield was recorded daily, but averaged per week because fat and protein contents in milk were measured weekly. The following formula was used to calculate FPCM (FAO, 2010):

$$
\operatorname{FPCM}(\mathrm{kg})=\text { raw milk }(\mathrm{kg}) \times[0.337+0.116
$$$$
\times \text { fat content }(\%)+0.06 \times \text { protein content }(\%)] \text {. }
$$ 
Editing DMI Records. Daily DMI records were averaged to weekly records to homogenize the data across experiments, resulting in 109,442 weekly records for DMI. Dry matter intake records of less than $5 \mathrm{~kg}$ per day were deleted, resulting in 109,052 weekly records from 3,954 cows. A second criterion was to select lactations of a cow with at least 5 weekly DMI records, resulting in 108,625 records from 3,853 cows. A third criterion was to select only experimental treatments with at least 5 animals, resulting in 108,516 records from 3,853 . Then, in the next step, only cows with at least $50 \%$ Holstein-Friesian genes were kept, resulting in 102,561 records from 3,653 cows. Later, data records from some experiments were deleted because the experiments had extreme residual variances $(\mathrm{SD}>5$ ) after a model was fitted, which adjusted for DIM, LW, milk, fat and protein yield, experimental treatment, breed, herd, and year-month effects, resulting in 98,375 records from 3,606 cows. Finally, only records from the first 3 lactations were kept, giving a total of 77,640 weekly DMI records from 3,188 cows (4,350 lactations in total). Analysis was performed on DMI, FPCM, and LW in the first lactation, referred to as DMI1, FPCM1, and LW1, respectively, and DMI, FPCM, and LW with information on more than 1 lactation (2 or 3 lactations), referred to as DMI3, FPCM3, and LW3, respectively. By considering DMI in lactations 1 to 3 as the same trait (DMI3), we assume a genetic correlation of 1 between lactations. A statistical description of the data per trait is summarized in Table 1.

\section{Genotypes}

A total of 1,496 cows were genotyped (1,421 of them with records on DMI) with 50K Illumina, 80K Geneseek, or 15K Eurogenomics SNP chips. The information from these 3 chips (missing genotypes and animals with $15 \mathrm{~K}$ and $50 \mathrm{~K}$ ) was imputed to a customized set of 76,439 SNP using Beagle 3 (Browning and Browning, 2009). After editing based on phenotype records of DMI, in terms of minimum and maximum, and the number of cows per experimental treatment, 1,313 animals were retained (all with DMI records). All 76,439 autosomal
SNP were used to calculate the GRM, according to Yang et al. (2010), whereas the inverse H-matrix (Aguilar et al., 2010; Christensen, 2012) was calculated using the Calc_grm program (Calus and Vandenplas, 2015) in combination with MiXBLUP software (ten Napel et al., 2016).

\section{Variance Component Estimation}

Two sets of trivariate analyses were performed separately to estimate the variance and covariance components between DMI1, FPCM1, and LW1, and between DMI3, FPCM3, and LW3, using ASReml4 (Gilmour et al., 2009). The model used to estimate the genetic parameters for DMI3, FPCM3, and LW3 was

$$
\begin{aligned}
y_{i j k l m n o p q r}= & \mu+E X P T R_{i}+H R_{j}+Y M_{k}+F R A C_{l} \cdot P A R_{m} \\
& +\sum_{n=0}^{3} A O C_{n} \Phi_{n} \cdot P A R_{m} \\
& +\sum_{n=0}^{5} D I M_{o} \Phi_{o} \cdot P A R_{m}+g_{p}+p e_{q}+e_{i j k l m n o p q r},
\end{aligned}
$$

where $y_{i j k l m n o p q r}$ is the phenotype for DMI3, FPCM3, and LW3; $\mu$ is the mean; EXPTR is the fixed effect $i$ for experimental treatment (515 classes); $H R$ is the fixed effect $j$ for herd (22 classes); YM is the fixed effect $k$ for year-month (272 classes); FRAC is the fixed effect of $l$ for fraction of Holstein-Friesian [defined as a percentage of Holstein-Friesian $(5 / 8,6 / 8,7 / 8$, and $8 / 8 ; 4$ classes)] by $m$ parity ( $P A R ; 3$ classes); $A O C$ is the fixed regression coefficient of the age of the cow measured in months by $P A R ; \Phi_{n}$ is the Legendre polynomial of the third order for $A O C ; D I M$ is the fixed regression coefficient of days in terms of milk as measured by PAR; $\Phi_{o}$ is the Legendre polynomial of the fifth order for DIM; $g$ is the additive genetic random effect $p$ distributed as $N\left(0, \mathbf{G} \sigma_{a}^{2}\right)$, in which $\mathbf{G}$ is the GRM in GBLUP, where $\sigma_{a}^{2}$ is the genetic variance; $p e$ is the permanent environmental random effect $q$ (both within and across parities) distributed as $N\left(0, \mathbf{I} \sigma_{c}^{2}\right)$, in which $\mathbf{I}$ is an identity matrix and $\sigma_{c}^{2}$ is the environmental variance; and $e$ is the residual random effect $r$ of $y_{i j k l m n o p q r}$ distributed as

Table 1. Descriptive statistics of DMI, fat- and protein-corrected milk (FPCM), and live weight (LW) in lactation 1 (DMI, FPCM1, and LW1, respectively), or over lactations 1 to 3 (DMI3, FPCM3, and LW3, respectively)

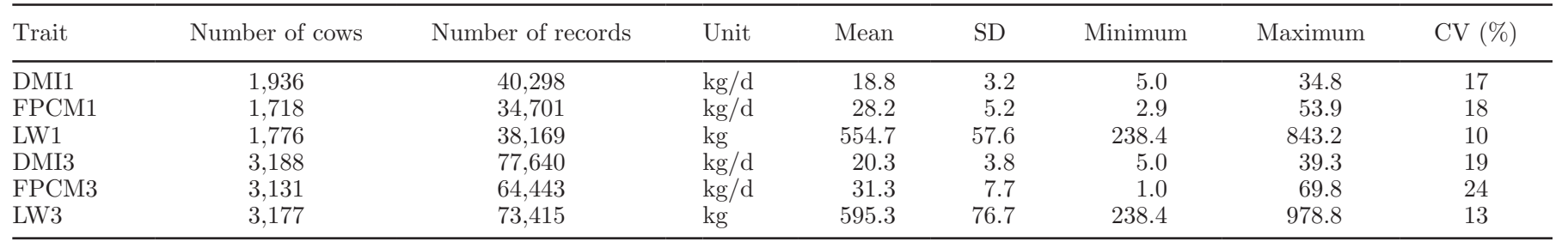


$N\left(0, \mathbf{I} \sigma_{e}^{2}\right)$, where $\sigma_{e}^{2}$ is the residual variance. The same model [1] was used to estimate genetic parameters for DMI1, FPCM1, and LW1, but excluding parity, as this analysis only used information from the first parity. This set of variance components was used for all models (BLUP, GBLUP, SS-GBLUP, and SSRR-BLUP) in the estimation of the EBV.

\section{Estimation of EBV}

The EBV for DMI1 and DMI3 for each animal were estimated using MiXBLUP software (ten Napel et al., 2016) with 4 different methods: BLUP, GBLUP, SS-GBLUP, and SSRR-BLUP. All 4 methods were implemented considering the same fixed effects and nongenetic random effects as in [1].

\section{BLUP, GBLUP, and SS-GBLUP}

The BLUP, GBLUP, and SS-GBLUP models were BLUP implementations described in model [2], using variance components as estimated with the model in [1], and the inverted relationship matrix $\mathbf{A}^{-1}, \mathbf{G}^{-1}$, or $\mathbf{H}^{-1}$ :

$$
\mathbf{y}=\mathbf{X} \boldsymbol{\beta}+\mathbf{Z}_{1} \mathbf{a}+\mathbf{Z}_{2} \mathbf{c}+\mathbf{e},
$$

where $\mathbf{y}$ is the vector of phenotypes; $\mathbf{X}$ and $\mathbf{Z}_{1}, \mathbf{Z}_{2}$ are incidence matrices relating observations with fixed, direct additive genetic, and permanent environmental effects; $\boldsymbol{\beta}$ is the vector of fixed effects; $\boldsymbol{a}$ is the vector of direct additive genetic effects; $\mathbf{c}$ is the vector of permanent environmental effects; and $\mathbf{e}$ is the vector of residual effects. Distributions of the random effects are $\operatorname{var}(\mathbf{a})=\mathbf{A} \sigma_{u}^{2}$ for the BLUP model using only pedigree (where $\mathbf{A}$ is the pedigree relationship matrix), $\operatorname{var}(\mathbf{a})=$ $\mathbf{G} \sigma_{u}^{2}$ for the GBLUP model (where $\mathbf{G}$ is the genomic relationship matrix), $\operatorname{var}(\mathbf{a})=\mathbf{H} \sigma_{u}^{2}$ for the SS-GBLUP model (where $\mathbf{H}$ is the combined pedigree and genomic relationship matrix), $\operatorname{var}(\mathbf{c})=\mathbf{I} \sigma_{c}^{2}$ (where $\mathbf{I}$ is identity matrix of order equal to the number of individuals with records), and $\operatorname{var}(\mathbf{e})=\mathbf{I} \sigma_{e}^{2}$ (where $\mathbf{I}$ is an identity matrix of an order equal to the number of observations); where $\sigma_{u}$ is the additive genomic variance, $\sigma_{c}$ is the permanent environmental variance, and $\sigma_{e}$ is the residual variance.

The G-matrix was built according to Yang et al. (2010) and calculated using Calc_grm (Calus and Vandenplas, 2015):

$$
\mathbf{G}=\frac{W W^{\prime}}{N},
$$

where $N$ is the number of SNP and $W$ contains the scaled and centered SNP genotypes for all animals at all loci, with elements computed for individual $j$ on locus $i$ as

$$
w_{i j}=\frac{\left(x_{i j}-2 p_{i}\right)}{\sqrt{2 p_{i}\left(1-p_{i}\right)}},
$$

where $w_{i j}$ is the genotype (scaled and centered) for animal $j$ at locus $i$, and $x_{i j}$ is the genotype for animal $j$ at locus $i$ before centering and scaling. Values for $p_{i}$ (i.e., the allele frequency at locus $i$ ) were calculated from the data. Unbiased estimates of genomic inbreeding coefficient for animal $\mathrm{j}$ with itself $\left(\mathbf{G}_{i j}\right)$ were computed to replace diagonal elements of $\mathbf{G}$ in Equation [3] (Yang et al., 2010) by

$$
\mathbf{G}_{j j}=1+\frac{1}{N} \Sigma_{i} \frac{x_{i j}{ }^{2}-\left(1+2 p_{i}\right) x_{i j}+2 p_{i}{ }^{2}}{2 p_{i}\left(1-p_{i}\right)} .
$$

The inverse of the H-matrix, $\mathbf{H}^{-1}$, is calculated with the following formula (Aguilar et al., 2010; Christensen and Lund, 2010):

$$
\mathbf{H}^{-1}=\mathbf{A}^{-1}+\left[\begin{array}{cc}
0 & 0 \\
0 & \lambda\left(\mathbf{G}^{-1}-\mathbf{A}_{22}^{-1}\right)
\end{array}\right],
$$

where $\mathbf{A}^{-1}$ is the inverse of the pedigree relationship matrix; $\mathbf{G}^{-1}$ is the inverse of the genomic relationship matrix; $\lambda$ is the value of 0.95 for lambda, and $\mathbf{A}_{22}^{-1}$ is the inverse of the pedigree relationship matrix among genotyped animals.

\section{SSRR-BLUP}

The SSRR-BLUP method is derived from a Bayesian regression method, which uses imputed SNP genotypes for nongenotyped animals, together with a residual genetic effect (Fernando et al., 2014). Each SNP genotype was centered by deducting the average value of the SNP genotypes across all genotyped individuals. The matrix $\mathbf{W}_{\text {non }}$ of imputed SNP genotypes was estimated from pedigree information and observed SNP genotypes in $\mathrm{W}_{\text {gen }}$ by solving $\mathbf{A}^{11} \mathrm{~W}_{\text {non }}=-\mathbf{A}^{12} \mathrm{~W}_{\text {gen }}$ (Fernando et al., 2014) for ancestors of genotyped individuals only. $\mathbf{A}^{11}$ and $\mathbf{A}^{12}$ are partitions of $\mathbf{A}^{-1}$ according to the individual being genotyped (2) or not (1). The SNP genotypes of nongenotyped individuals that were not ancestor of a genotyped individual were imputed by taking the parental average SNP genotypes if parents 
Table 2. Numbers of sires (of validation cows), validation and reference cows, validation and reference records per cross-validation group, and total number of sires, cows, and records available across validation groups

\begin{tabular}{|c|c|c|c|c|c|}
\hline \multirow[b]{2}{*}{ Item $^{1}$} & \multicolumn{4}{|c|}{ Cross-validation group } & \multirow[b]{2}{*}{ Total $^{2}$} \\
\hline & 1 & 2 & 3 & 4 & \\
\hline Sires of validation cows & 68 & 74 & 70 & 73 & 285 \\
\hline Validation cows & 352 & 303 & 323 & 335 & 1,313 \\
\hline Reference cows & 961 & 1,010 & 990 & 978 & 1,313 \\
\hline Validation DMI1 records GBLUP & 6,455 & 3,677 & 4,486 & 5,982 & 20,600 \\
\hline Reference DMI1 records GBLUP & 14,145 & 16,923 & 16,114 & 14,618 & 20,600 \\
\hline Validation DMI1 records SS-GBLUP ${ }^{3}$ & 9,013 & 7,068 & 7,974 & 8,578 & 32,627 \\
\hline Reference DMI1 records SS-GBLUP ${ }^{3}$ & 23,614 & 25,559 & 24,653 & 24,049 & 32,627 \\
\hline Validation DMI3 records GBLUP ${ }^{3}$ & 6,455 & 3,677 & 4,486 & 5,982 & 20,600 \\
\hline Reference DMI3 records GBLUP & 33,843 & 36,621 & 35,812 & 34,314 & 40,298 \\
\hline Validation DMI3 records SS-GBLUP ${ }^{3}$ & 9,013 & 7,068 & 7,974 & 8,578 & 32,627 \\
\hline Reference DMI3 records SS-GBLUP ${ }^{3}$ & 68,627 & 70,572 & 69,666 & 69,062 & 77,640 \\
\hline
\end{tabular}

were known or set to zero if parents were unknown. After imputation, the average value of each SNP genotype across all genotyped individuals was added to the imputed SNP genotypes.

The SSRR-BLUP model used to calculate the EBV can be represented as follows:

$$
\mathbf{y}=\mathbf{X} \boldsymbol{\beta}+\left[\begin{array}{c}
\mathbf{W}_{\text {non }} \\
\mathbf{W}_{\text {gen }}
\end{array}\right] \boldsymbol{\alpha}+\delta \mathbf{Z}_{1} \varepsilon+\mathbf{Z}_{2} \mathbf{c}+\mathbf{e},
$$

where $\mathbf{y}$ is the vector of phenotypes; $\mathbf{X}$ is the incidence matrix of the fixed effects; $\mathbf{W}_{\text {non }}$ is the regression design matrix with imputed SNP genotypes; $\mathbf{W}_{\text {gen }}$ is the regression design matrix with observed SNP genotypes; $\mathbf{Z}_{1}, \mathbf{Z}_{2}$ are incidence matrices relating observations with genetic and permanent environmental effects; $\boldsymbol{\beta}$ is the vector of fixed effects; $\boldsymbol{\alpha}$ is the vector of SNP regression coefficients; $\boldsymbol{\delta}$ is a vector with 1 for nongenotyped and 0 for genotyped animals; $\mathbf{c}$ is the vector of permanent environmental effects; $\varepsilon$ is the vector with residual genetic solutions, in addition to the genetic effect explained by the imputed SNP genotypes $\left(\mathbf{W}_{\text {non }} \times \boldsymbol{\alpha}\right)$; and $\mathbf{e}$ is the vector of random residual effects.

The SNP variances were calculated with the following formula for SNP for noncentered SNP genotypes:

$$
V_{\mathrm{SNP}}=\frac{\sigma_{u}^{2}}{\sum_{i=1}^{n_{\text {inf }}} 2 \times p_{i} \times\left(1-p_{i}\right)},
$$

where $V_{\mathrm{SNP}}$ is the SNP variance; $\sigma_{u}^{2}$ is the genetic variance; $n_{\text {inf }}$ is the number of informative SNP (all 3 genotypes present); and $p_{i}$ is the allele frequency of SNP $i$.
It is important to note that, for the GBLUP method, only animals with genotypes and phenotypes were used, meaning that fewer data were used (Table 2) compared with BLUP, SS-GBLUP, and SSRR-BLUP, where both genotyped and nongenotyped animals with phenotypes were included.

\section{Validation}

Cross-Validation. The models were validated using cross-validation, where the population of genotyped individuals was divided into 4 subsets, making sure that all paternal half-sibs were in the same group. The assignment to the groups was made by sire, using stratified random sampling, which was undertaken in 2 steps: first, the sires of genotyped animals were sorted from the highest to the fewest daughters. Then, from every set of 4 subsequent sires, one sire was randomly allocated to one of the 4 groups. Thus, the 4 validation groups were equivalent in terms of the proportion of sires, number of cows with genotypes, and the total number of records. The number of animals in the crossvalidation groups is shown in Table 2 . For each of the validation groups, EBV were predicted after excluding the respective phenotypes from the analysis, using phenotypes of the other 3 groups only.

Calculation of Accuracies. The accuracies were calculated via cross-validation per scenario as the correlation between the adjusted phenotype for DMI1 or DMI3 and the EBV for DMI1 or DMI3. Adjusted phenotypes for DMI1 and DMI3 were calculated per animal in ASReml4 (Gilmour et al., 2009) as the solutions of a model using all fixed effects in the model in [1], but excluding the genetic animal effect. The idea 
behind this was to have a single adjusted phenotype per animal, instead of multiple adjusted phenotypes. The accuracies were divided by the approximated accuracy of the adjusted phenotypes, computed with the formula (Falconer and Mackay., 1996):

$$
\sqrt{\frac{n \mathrm{~h}^{2}}{1+(n-1) r e p}},
$$

where $n$ is the average number of repeated records per animal per cross-validation group (21 for DMI1 and 25 for DMI3); $h^{2}$ is heritability of the trait (0.18 for DMI1 and 0.17 for DMI3; see Results and Discussion section); and rep is the repeatability of the trait calculated as the ratio between the genetic and permanent environmental variance between the phenotypic variance $(0.49$ for DMI1 and 0.44 DMI3; Table 4). Accuracies were calculated for each validation group $(1,2,3,4)$ and averaged across validation groups. Standard errors of accuracies per scenario were defined as the SD of the accuracy across all validation groups divided by the square root of the number of validation groups. Slopes of regression (linear regression coefficients) of adjusted phenotypes (DMI1 and DMI3) on EBV were calculated per method and per scenario as a measure of the bias in terms of the variance in EBV.

\section{Validation Scenarios}

Four validation scenarios were performed: (1) using information sources from lactation 1 to validate in DMI1; (2) using information sources for all 3 lactations to validate in DMI1; (3) using information sources from lactation 1 to validate in DMI3; and (4) using information sources from the 3 lactations to validate in DMI3. To better illustrate this idea, a scheme is presented in Table 3. For each method, a set of 6 analyses was performed (Table 3); 3 used information from lactation 1 , whereas the other 3 used information from lactations 1 to 3 , each one with 3 variants depending on the information source used. Thus, the 6 scenarios were (1) DMI1; (2) FPCM1 and LW1; (3) DMI1, FPCM1, and LW1; (4) DMI3; (5) FPCM3 and LW3; and (6) DMI3, FPCM3, and LW3.

\section{RESULTS AND DISCUSSION}

\section{Estimated Genetic Parameters}

The data set contained 77,640 weekly records, recorded during 4,350 lactations involving 3,188 cows (Table 1). Compared with most studies, this is a relatively large data set for estimating genetic parameters for DMI as some reviews mentioned (Korver, 1988; Veerkamp, 1998; Berry and Crowley, 2013). The mean for DMI1 was $18.8 \mathrm{~kg} / \mathrm{d}$, whereas the mean for DMI3 was higher $(22.32 \mathrm{~kg} / \mathrm{d})$. The mean for FPCM1 and FPCM3 were 28.19 and $31.26 \mathrm{~kg} / \mathrm{d}$, respectively. The mean for LW1 and LW3 was 554.7 and $595.3 \mathrm{~kg}$, respectively. The SD of the traits including the first 3 lactations was always higher than those including only the first lactation.

Genetic variances for DMI1, FPCM1, and LW1 (Table 4) were $0.91(\mathrm{~kg} / \mathrm{d})^{2}, 4.01(\mathrm{~kg} / \mathrm{d})^{2}$, and 1,009 $\mathrm{kg}^{2}$, whereas for DMI3, FPCM3, and LW3 were 1.06 $(\mathrm{kg} / \mathrm{d})^{2}, 5.94(\mathrm{~kg} / \mathrm{d})^{2}$, and $1,254 \mathrm{~kg}^{2}$, respectively. For DMI and FPCM, the permanent environmental variances were higher than the genetic variances, whereas, for $\mathrm{LW}$, the permanent environmental variance was lower than the genetic variance. In the case of DMI and FPCM, the higher permanent environmental variances compared with genetic variances could be due to the repeated measures of these traits that are catching part of the total variation that is not genetic. Estimated heritabilities were 0.18 and 0.17 for DMI1 and DMI3, 0.23 and 0.24 for FPCM1 and FPCM3, and 0.47 and 0.43 for LW1 and LW3, respectively. These estimated heritabilities of DMI1 and DMI3 were within the range (0.11 to 0.35 ) of previous studies presenting heritabilities (Veerkamp, 1998; Veerkamp and Thompson, 1999; Berry et al., 2007; Vallimont et al., 2010, 2011; Williams et al., 2011; Spurlock et al., 2012). Estimated heritabilities of FPCM in the current study were slightly lower than previously reported heritabilities ranging between 0.27 and 0.47 (Van Arendonk et al., 1991; Veerkamp et al., 2012; Pszczola et al., 2013; Lassen et al., 2016); however, the repeatabilities were high (between 0.63 and 0.66; Table 4), meaning that a big portion of the variance was explained by the permanent environmental instead of the genetic variance. Estimated heritabilities for LW in the current study were in the range of estimates in the literature ranging from 0.43 to 0.65 (Veerkamp and Brotherstone, 1997; Koenen and Veerkamp, 1998; Berry et al., 2003; Muller et al., 2006; Dechow et al., 2010).

Table 3. Validation traits and scenarios per method used (BLUP genomic BLUP, single-step genomic BLUP, and single-step ridgeregression BLUP)

\begin{tabular}{ll}
\hline Validation trait & Reference traits per scenario $^{1}$ \\
\hline DMI1 or DMI3 & (1a) DMI1 \\
& (1b) FPCM1, LW1 \\
& (1c) DMI1, FPCM1, LW1 \\
& (2a) DMI3 \\
& (2b) FPCM3, LW3 \\
& (2c) DMI3, FPCM3, LW3 \\
\hline
\end{tabular}

${ }^{1} \mathrm{FPCM}=$ fat- and protein-corrected milk; LW = live weight. $1=$ lactation $1 ; 3=$ lactations 1 to 3 . 
Table 4. Estimated genetic $\left(\sigma_{a}^{2}\right)$, permanent environmental $\left(\sigma_{p e}^{2}\right), \operatorname{residual}\left(\sigma_{e}^{2}\right)$, and phenotypic $\left(\sigma_{p}^{2}\right)$ variances, heritabilities (on diagonal, bold), and genetic $\left(\mathrm{r}_{\mathrm{a}}\right.$; below diagonal) and phenotypic correlations $\left(\mathrm{r}_{\mathrm{p}}\right.$; above diagonal) using the genomic BLUP method for traits in lactation 1 [DMI1, fat- and protein-corrected milk (FPCM)1, live weight (LW)1], and traits in the 3 lactations (DMI3, FPCM3, and LW3) used to estimate the EBV

\begin{tabular}{llcccccccc}
\hline \multirow{2}{*}{ Lactation } & Trait & \multicolumn{2}{c}{$\sigma_{a}^{2}$} & $\sigma_{p e}^{2}$ & $\sigma_{e}^{2}$ & $\sigma_{p}^{2}$ & $r e p^{1}$ & \multicolumn{3}{c}{$\mathrm{h}^{2}, \mathrm{r}_{\mathrm{a}}$ and $\mathrm{r}_{\mathrm{p}}$} \\
\hline 1 & DMI1 & 0.91 & 1.54 & 2.56 & 5.01 & 0.49 & $\mathbf{0 . 1 8}$ & 0.50 & 0.30 \\
& FPCM1 & 4.01 & 7.76 & 5.96 & 17.73 & 0.66 & 0.65 & $\mathbf{0 . 2 3}$ & 0.06 \\
\multirow{4}{*}{$1-3$} & LW1 & 1,009 & 754.6 & 398.1 & $2,161.7$ & 0.82 & 0.68 & 0.07 & $\mathbf{0 . 4 7}$ \\
& DMI3 & 1.06 & 1.61 & 3.42 & 6.09 & 0.44 & $\mathbf{0 . 1 7}$ & 0.48 & 0.24 \\
& FPCM3 & 5.94 & 9.90 & 9.20 & 25.04 & 0.63 & 0.59 & $\mathbf{0 . 2 4}$ & 0.03 \\
& LW3 & 1,254 & 1,004 & 658.8 & $2,916.8$ & 0.77 & 0.43 & -0.02 & $\mathbf{0 . 4 3}$ \\
\hline
\end{tabular}

${ }^{1}$ rep $=$ repeatability of the trait calculated as the ratio between the genetic and permanent environmental variance between the phenotypic variance.

Moderate to high (0.4 to 0.8$)$ genetic correlations between DMI and predictor traits (i.e., FPCM and LW) have been estimated in several studies (Veerkamp and Brotherstone, 1997; Cooper et al., 2010; Vallimont et al., 2011; Berry and Crowley, 2013). In the current study, the genetic correlation of DMI with FPCM1 was 0.65 , and 0.68 with LW1, whereas the genetic correlation between DMI3 and FPCM3 was 0.59 and between DMI3 and FPCM3 was 0.43. With these correlations, we can conclude that it is possible to estimate EBV for DMI by using predictor traits, such as FPCM and LW, as they explained a large proportion of the variance in DMI. However, it is important to realize that the highest accuracy of prediction of DMI will be determined by the size of the genetic correlation.

\section{Accuracies of EBV for DMI}

Accuracies of EBV for DMI1 and DMI3 per scenario and method (BLUP, GBLUP, SS-GBLUP, and SSRR-BLUP) are presented in Table 5. In general, the accuracies of the EBV for DMI estimated in this study were in agreement with average accuracies across validation sets in other studies, ranging between 0.20 and 0.45 (Pszczola et al., 2013; Pryce et al., 2014; de Haas et al., 2015). However, when the predictor traits were recorded in both the reference population and the validation population, higher accuracies were achieved, ranging from 0.47 to 0.63 (Pszczola et al., 2013).

\section{Across Lactations}

As DMI varies between lactations (Berry et al., 2006), it is of importance to know whether using traits, which contain information about the 3 lactations in the reference population, would result in higher accuracies than using traits that contain information about lactation 1. The accuracies of EBV for DMI1 and DMI3, as estimated with a reference set with traits containing information on only lactation 1 , ranged from 0.14 to 0.30 (SE between 0.03 and 0.09), whereas the accuracies of EBV for DMI1 and DMI3, as estimated with

Table 5. Accuracies of prediction of EBV averaged across validation groups (SE in parentheses), for validation traits DMI1 (lactation 1 only) and DMI3 (lactations 1-3) using different combinations of reference traits and method [BLUP, genomic BLUP (GBLUP), single-step GBLUP (SS-GBLUP), and single-step ridge-regression BLUP (SSRR-BLUP)]

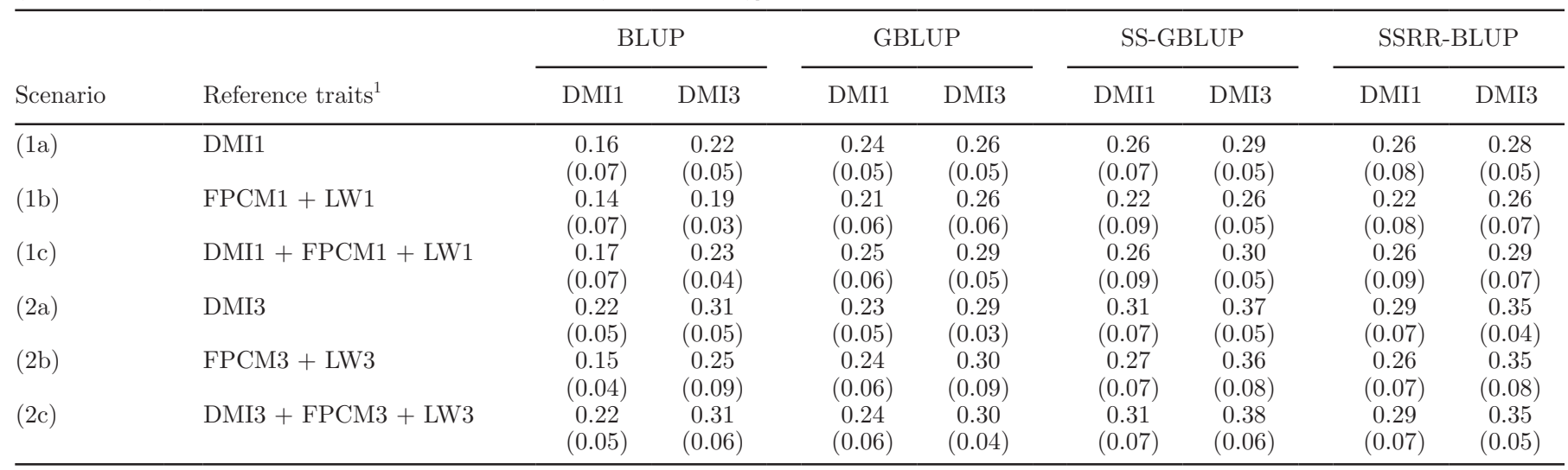

${ }^{1} \mathrm{FPCM}=$ fat- and protein-corrected milk; LW = live weight. 1 = lactation $1 ; 3=$ lactations 1 to 3. 
a reference set with traits containing information on all 3 lactations, ranged from 0.15 to 0.38 (SE between 0.04 and 0.09 ; Table 5). With this information we can infer that the results between those 2 reference sets are not significantly different. Therefore, the accuracies of EBV based on a reference set with traits containing information on all 3 lactations (DMI3, FPCM3, and LW3), regardless of the validation trait (DMI1 or DMI3), were higher, compared with reference sets with traits only containing information about lactation 1 (DMI1, FPCM1, and LW1). In addition, in the current study, the correlation between DMI1 and DMI3 was 0.6 , showing a difference between DMI in first parity cows and DMI in later parities, as has been reported previously (Berry et al., 2006). This correlation implies that DMI1 and DMI3 are different traits, as traits with a genetic correlation below 0.8 can be considered to be different traits (Robertson, 1959). These results, however, demonstrate that the assumption that DMI in different lactations is the same trait, is beneficial for genomic prediction, and at least approximately correct, and could have a positive effect on the accuracy of a multicountry reference set, as de Haas et al. (2012) reported.

\section{Across Scenarios}

Accuracies of estimated EBV for DMI1 and DMI3 were generally the same or slightly higher when DMI was included as a trait in the reference population (scenario a: only DMI; scenario c: DMI, FPCM, and LW) than when DMI was not included as a reference population trait and when the reference population contained only FPCM and LW (scenario b). This is more evident in DMI1 than in DMI3 (Table 5). Additionally, there were either no or slight differences in accuracies between scenarios a and c, meaning that the value of adding additional information about predictor traits, when the DMI is already recorded in the reference population on the same animals, is limited. However, this result could be affected by many factors such as the size and structure of the reference population, and its relationship with the validation set. Besides, in this study, the animals in the reference population were selected based on whether they had DMI records (not based on animals with predictor traits); probably if it were not the case, the results would be different. When only using predictor traits in the reference population, the accuracies of estimated EBV for DMI1 and DMI3 were lower than in the scenarios using DMI. The difference was not large, indicating that, in absence of DMI records, FPCM and LW could still predict a large and useful part of DMI. This was also the case with the accuracies obtained with the BLUP method. These results are in agreement with Pszczola et al. (2013), in which no benefit was observed when adding FPCM to DMI in the reference population, whereas only a slight increase in accuracy (0.02) was observed when adding LW to DMI and FPCM in the reference population.

\section{Across Methods}

The accuracies of EBV using BLUP were the lowest accuracies obtained, ranging between 0.14 and 0.31 , compared with the other 3 methods that included genomic information. Accuracies of EBV for DMI1 were lowest with the GBLUP method, ranging between 0.21 and 0.25 , followed by the SS-GBLUP method (between 0.22 and 0.31 ) and the SS-RRBLUP method (between 0.22 and 0.29 ). With this information we can infer that the results between SS-GBLUP and SSRR-BLUP are not significantly different. Likewise, accuracies of EBV of DMI3 were lowest with the GBLUP method, ranging from 0.26 to 0.30 , followed by the SS-GBLUP method (between 0.26 and 0.38) and the SSRR-BLUP method (between 0.26 and 0.35). These results are in line with the results of other studies, which concluded that applying the single-step methods utilizing all the data of genotyped and nongenotyped animals resulted in more accurate EBV than applying the GBLUP method, for example, for 16 economic important traits, such as fertility, milk, and longevity in Nordic Red cattle (Gao et al., 2012; Su et al., 2012). Additionally, SS-GBLUP has proven to be more accurate and simpler than multistep methods (Christensen, 2012) and is currently broadly used for genetic evaluations in dairy and beef cattle. In the current study, applying the SS-GBLUP method and the SSRR-BLUP method resulted in similar accuracies for EBV of DMI1, when information on lactation 1 was used. However, when information from 3 lactations was used in the reference population, slightly higher accuracies were estimated for the SS-GBLUP method, compared with SSRR-BLUP. The generally similar results between SS-GBLUP and SSRR-BLUP are not surprising, as both methods are expected to be equivalent (Fernando et al., 2014).

\section{Correlations Between EBV Per Method}

In Table 6, average pairwise correlations between EBV estimated by each of the 4 methods (BLUP, GBLUP, SS-GBLUP, and SSRR-BLUP) per scenario are presented. The lowest correlations were between EBV obtained by BLUP and GBLUP method ranging between 0.46 and 0.52 . This is probably because those methods are further apart than the rest, as BLUP 
only used pedigree information through the pedigree relationship matrix and GBLUP used genomic information through the genomic relationship matrix. The correlations between EBV obtained by BLUP with SSGBLUP method were slightly higher than the correlations between BLUP and SSRR-BLUP. Further, the correlations between EBV estimated with GBLUP and SS-GBLUP ranged between 0.74 to 0.78 and between GBLUP and SSRR-BLUP (0.80 to 0.82 ) were presented, showing that EBV of SSRR-BLUP are slightly more similar to GBLUP than those from SS-GBLUP; however, this relationship between those models remains unclear. Finally, the correlations between EBV estimated with SS-GBLUP and SSRR-BLUP were between 0.94 to 0.98 across scenarios, showing that these methods indeed are very similar (Table 6 ). The main advantage of SSRR-BLUP, compared with SS-GBLUP, is that it can handle more genotyped animals because it avoids the calculation of the G-matrix and its inverse. For the data used here, the current implementation of SSRR-BLUP in the MiXBLUP software (ten Napel et al., 2016) required a larger number of iterations to converge compared with SS-GBLUP, such that SSRRBLUP required substantially more computing time than SS-GBLUP. Therefore, SS-GBLUP was faster given the lower number of iterations needed to converge for these data because the number of genotyped animals (1,313), which determines the size of the GRM, is relatively small. However, calculating the G-inverse would be challenging in a scenario with hundreds of thousands of genotyped animals. Therefore, it is recommended that both methods are tested with a larger number of genotyped animals to study the advantages and disadvantages of SSRR-BLUP compared with SSGBLUP. Future developments will likely help to further improve the performance of SSRR-BLUP in terms of convergence. Recent developments suggest that mixed model equations for SSRR-BLUP can be rewritten to reduce the number of iterations required (Fernando and Narayana, 2016). However, SSRR-BLUP may yield a quasi-singular system of equations and may therefore be suboptimal in terms of expected convergence $r$.

\section{Slopes}

Slopes of regression of adjusted phenotypes for DMI1 and DMI3 on EBV for DMI1 and DMI3 per scenario and method (BLUP, GBLUP, SS-GBLUP, and SSRRBLUP) are presented in Table 7 . These regression slopes can be seen as a measure of bias in terms of the variance in EBV. Slopes smaller than 1 indicate an overestimation of the variance in EBV, whereas slopes larger than 1 indicate an underestimation of the variance in EBV. In general, in the current study, the slopes were smaller

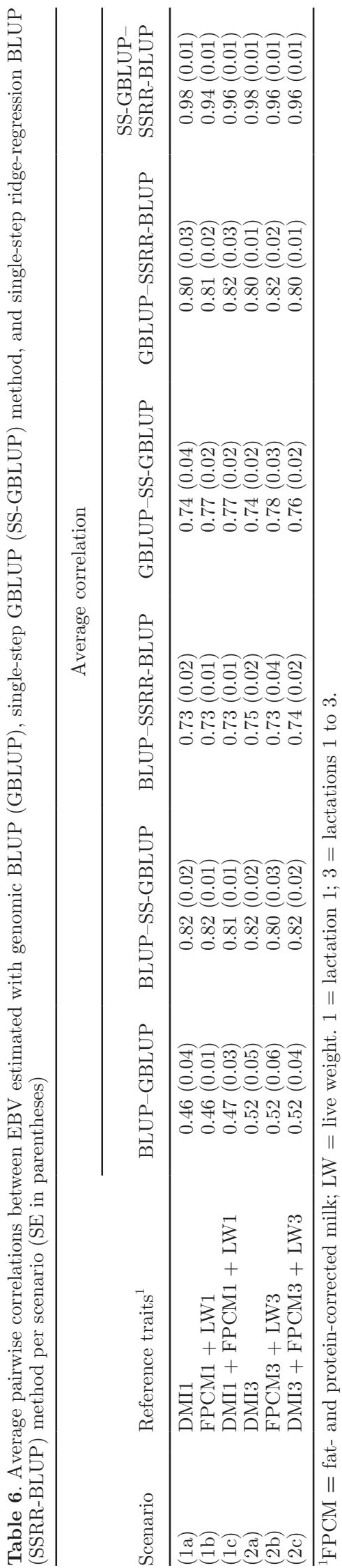

Journal of Dairy Science Vol. 100 No. 11, 2017 
Table 7. Average slope of regression for EBV on adjusted phenotypes across validation groups (SE in parentheses), for validation traits DMI1 (lactation 1 only) and DMI3 (lactations 1-3) using different combinations of reference traits and method [BLUP, genomic BLUP (GBLUP), single-step GBLUP (SS-GBLUP), and single-step ridge-regression BLUP (SSRR-BLUP)]

\begin{tabular}{|c|c|c|c|c|c|c|c|c|c|}
\hline Scenario & Reference traits ${ }^{1}$ & \multicolumn{2}{|c|}{ BLUP } & \multicolumn{2}{|c|}{ GBLUP } & \multicolumn{2}{|c|}{ SS-GBLUP } & \multicolumn{2}{|c|}{ SSRR-BLUP } \\
\hline (1a) & DMI1 & $\begin{array}{c}0.37 \\
(0.18)\end{array}$ & $\begin{array}{c}0.67 \\
(0.17)\end{array}$ & $\begin{array}{c}0.63 \\
(0.10)\end{array}$ & $\begin{array}{c}0.82 \\
(0.16)\end{array}$ & $\begin{array}{c}0.56 \\
(0.17)\end{array}$ & $\begin{array}{c}0.78 \\
(0.18)\end{array}$ & $\begin{array}{c}0.55 \\
(0.17)\end{array}$ & $\begin{array}{c}0.76 \\
(0.15)\end{array}$ \\
\hline (1b) & FPCM1 + LW1 & $\begin{array}{c}0.36 \\
(0.17)\end{array}$ & $\begin{array}{c}0.60 \\
(0.12)\end{array}$ & $\begin{array}{c}0.51 \\
(0.15)\end{array}$ & $\begin{array}{c}0.78 \\
(0.18)\end{array}$ & $\begin{array}{c}0.45 \\
(0.23)\end{array}$ & $\begin{array}{c}0.71 \\
(0.15)\end{array}$ & $\begin{array}{c}0.44 \\
(0.26)\end{array}$ & $\begin{array}{c}0.74 \\
(0.20)\end{array}$ \\
\hline (1c) & DMI1 + FPCM1 + LW1 & $\begin{array}{c}0.41 \\
(0.18)\end{array}$ & $\begin{array}{c}0.70 \\
(0.14)\end{array}$ & $\begin{array}{c}0.62 \\
(0.15)\end{array}$ & $\begin{array}{c}0.87 \\
(0.16)\end{array}$ & $\begin{array}{c}0.53 \\
(0.21)\end{array}$ & $\begin{array}{c}0.79 \\
(0.16)\end{array}$ & $\begin{array}{c}0.55 \\
(0.24)\end{array}$ & $\begin{array}{c}0.83 \\
(0.19)\end{array}$ \\
\hline$(2 \mathrm{~b})$ & FPCM3 + LW3 & $\begin{array}{c}0.42 \\
(0.09)\end{array}$ & $\begin{array}{c}0.83 \\
(0.26)\end{array}$ & $\begin{array}{c}0.58 \\
(0.14)\end{array}$ & $\begin{array}{c}0.88 \\
(0.26)\end{array}$ & $\begin{array}{c}0.60 \\
(0.15)\end{array}$ & $\begin{array}{l}1.01 \\
(0.21)\end{array}$ & $\begin{array}{c}0.60 \\
(0.16)\end{array}$ & $\begin{array}{c}1.01 \\
(0.23)\end{array}$ \\
\hline (2c) & DMI3 + FPCM3 + LW3 & $\begin{array}{c}0.41 \\
(0.08)\end{array}$ & $\begin{array}{c}0.72 \\
(0.15)\end{array}$ & $\begin{array}{r}0.43 \\
(0.11\end{array}$ & $\begin{array}{c}0.65 \\
(0.08)\end{array}$ & $\begin{array}{c}0.50 \\
(0.11)\end{array}$ & $\begin{array}{c}0.77 \\
(0.14)\end{array}$ & $\begin{array}{c}0.50 \\
(0.13)\end{array}$ & $\begin{array}{c}0.75 \\
(0.13)\end{array}$ \\
\hline
\end{tabular}

${ }^{1} \mathrm{FPCM}=$ fat- and protein-corrected milk; $\mathrm{LW}=$ live weight. 1 = lactation $1 ; 3=$ lactations 1 to 3.

than 1 across methods and scenarios, suggesting that the variance of EBV was somewhat inflated.

Across Traits (DMI1 and DMI3). Slopes were closer to 1 for the validation trait DMI3 than for the validation trait DMI1 for all scenarios and methods, showing an overestimation of the variance in EBV when using DMI1 as validation trait compared with DMI3.

Across Scenarios. When using information from lactation 1, slopes were closer to 1 when DMI1 (scenarios 1a and 1c) was included in the reference population, compared with when only predictor traits were included in the reference population. These results are in agreement with Pszczola et al. (2013) that reported slopes closer to 1, when DMI, FPCM, and LW were added to the reference population to predict DMI, showing that, when information on additional traits was added to both reference and validation population, the variance in EBV was unbiased. However, this was not the case for reference traits that included 3 lactations, where scenario 2b (FPCM3 and LW3) had greater slopes (in some cases close to 1) compared with scenarios 2a and 2c. The mechanism behind these results is not clear yet.

Across Methods. The BLUP method had the lowest slope (0.36) compared with the other methods, meaning that the variance in the EBV were severely overestimated in this method. The slopes for GBLUP varied between 0.41 and 0.88 , whereas the slopes varied between 0.44 and 1.01 for SS-GBLUP and SSRR-BLUP. This suggests that SS-GBLUP and SSRR-BLUP yielded less bias in EBV variance compared with GBLUP. Finally, slopes of 1 were obtained in both SS-GBLUP and SSRR-BLUP methods only for validation trait DMI3 in scenario $2 \mathrm{~b}$, showing the variance in $\mathrm{EBV}$ was least biased when FPCM and LW information was included on all 3 lactations.

\section{CONCLUSIONS}

In the present study, applying SS-GBLUP and SSRRBLUP methods to estimate EBV for DMI resulted in higher accuracies, compared with applying the BLUP and GBLUP methods, because SS-GBLUP and SSRRBLUP allowed for the incorporation of additional information on phenotypic records of nongenotyped animals. The accuracies of EBV for DMI were similar in the SS-GBLUP and SSRR-BLUP methods, proving that both methods perform in a similar fashion. Thus, SSRR-BLUP could be used instead of SS-GBLUP when the number of genotyped animals is large, avoiding the construction of the $\mathbf{G}$ matrix and computing its inverse, while yielding similar results. Furthermore, adding more information about DMI from different lactations in the reference population gave a higher degree of accuracies, compared with when information about lactation 1 alone was included. In general, adding predictor traits to the reference population did not improve the accuracy concerning DMI when DMI was already included. However, in the absence of DMI records, having records on FPCM and LW from different lactations for the reference population is an efficient way to obtain EBV for DMI with a useful degree of accuracy.

\section{ACKNOWLEDGMENTS}

Data from the national database were provided by CRV (Arnhem, the Netherlands). The first author thanks the National Council on Science and Technology of Mexico (CONACYT, Mexico) for its financial support to study for a $\mathrm{PhD}$ at Wageningen University, and to the National Research Institute of Forestry, 
Agriculture and Livestock (INIFAP, Mexico) for promoting the professional development of the researchers. Mathijs van Pelt, Ina Hulsegge, and Ghyslaine Schopen (Wageningen Livestock Research, Wageningen, the Netherlands) are kindly acknowledged for their help regarding the realization of this study.

\section{REFERENCES}

Aguilar, I., I. Misztal, D. L. Johnson, A. Legarra, S. Tsuruta, and T. J. Lawlor. 2010. Hot topic: A unified approach to utilize phenotypic, full pedigree, and genomic information for genetic evaluation of Holstein final score. J. Dairy Sci. 93:743-752.

Beerda, B., W. Ouweltjes, L. B. J. Sebek, J. J. Windig, and R. F. Veerkamp. 2007. Effects of genotype by environment interactions on milk yield, energy balance, and protein balance. J. Dairy Sci. 90:219-228.

Berry, D. P., F. Buckley, P. Dillon, R. D. Evans, M. Rath, and R. F. Veerkamp. 2003. Genetic parameters for body condition score, body weight, milk yield, and fertility estimated using random regression models. J. Dairy Sci. 86:3704-3717.

Berry, D. P., and J. J. Crowley. 2013. Cell Biology Symposium: Genetics of feed efficiency in dairy and beef cattle. J. Anim. Sci. 91:1594-1613.

Berry, D. P., B. Horan, M. O’Donovan, F. Buckley, E. Kennedy, M. McEvoy, and P. Dillon. 2007. Genetics of grass dry matter intake, energy balance, and digestibility in grazing Irish dairy cows. J. Dairy Sci. 90:4835-4845.

Berry, D. P., R. F. Veerkamp, and P. Dillon. 2006. Phenotypic profiles for body weight, body condition score, energy intake, and energy balance across different parities and concentrate feeding levels. Livest. Sci. 104:1-12.

Browning, B. L., and S. R. Browning. 2009. A unified approach to genotype imputation and haplotype-phase inference for large data sets of trios and unrelated individuals. Am. J. Hum. Genet. 84:210-223.

Calus, M. P. L., and J. Vandenplas. 2015. Calc_grm-A program to compute pedigree, genomic, and combined relationship matrices. ABGC, Wageningen UR Livestock Research. Wageningen, the Netherlands.

Christensen, O. F. 2012. Compatibility of pedigree-based and marker-based relationship matrices for single-step genetic evaluation. Genet. Sel. Evol. 44:37.

Christensen, O. F., and M. S. Lund. 2010. Genomic prediction when some animals are not genotyped. Genet. Sel. Evol. 42:2.

Cooper, A. J., C. L. Ferrell, L. V. Cundiff, and L. D. Van Vleck. 2010. Prediction of genetic values for feed intake from individual body weight gain and total feed intake of the pen. J. Anim. Sci. 88:1967-1972.

de Haas, Y., M. P. L. Calus, R. F. Veerkamp, E. Wall, M. P. Coffey, H. D. Daetwyler, B. J. Hayes, and J. E. Pryce. 2012. Improved accuracy of genomic prediction for dry matter intake of dairy cattle from combined European and Australian data sets. J. Dairy Sci. 95:6103-6112.

de Haas, Y., J. E. Pryce, M. P. L. Calus, E. Wall, D. P. Berry, P. Lovendahl, N. Krattenmacher, F. Miglior, K. Weigel, D. Spurlock, K. A. Macdonald, B. Hulsegge, and R. F. Veerkamp. 2015. Genomic prediction of dry matter intake in dairy cattle from an international data set consisting of research herds in Europe, North America, and Australasia. J. Dairy Sci. 98:6522-6534.

Dechow, C. D., J. Vallimont, M. D. Dekleva, J. M. Daubert, and J. W. Blum. 2010. Genetic correlations of gross feed efficiency with yield, body weight, body condition score, and energy balance in dairy cattle. J. Dairy Sci. 93:184-184.

European Union. 2011. European Commission of Agricultural and Rural Development. Farm Economics Brief. No. 2 Production costs overview. Accessed Nov. 7, 2016. http://ec.europa.eu/agriculture/ rica/pdf/Brief201102.pdf.
Falconer, D. S., and T. F. C. Mackay. 1996. Introduction to Quantitative Genetics. 4th ed. Pearson, Prentice Hall, Upper Saddle River, NJ.

FAO. 2010. Greenhouse gas emissions from the dairy sector. Food and Agriculture Organization of the United Nations, Rome, Italy.

Fernando, N., and M. Narayana. 2016. A comprehensive two dimensional Computational Fluid Dynamics model for an updraft biomass gasifier. Renew. Energy 99:698-710.

Fernando, R. L., J. C. M. Dekkers, and D. J. Garrick. 2014. A class of Bayesian methods to combine large numbers of genotyped and non-genotyped animals for whole-genome analyses. Genet. Sel. Evol. 46:50.

Gao, H. D., O. F. Christensen, P. Madsen, U. S. Nielsen, Y. Zhang, M. S. Lund, and G. S. Su. 2012. Comparison on genomic predictions using three GBLUP methods and two single-step blending methods in the Nordic Holstein population. Genet. Sel. Evol. 44:8.

Gilmour, A. R., B. J. Gogel, B. R. Cullis, and R. Thompson. 2009 ASREML User Guide Release 3.0 VSN International Ltd., Hemel Hempstead, UK.

Koenen, E. P. C., and R. F. Veerkamp. 1998. Genetic covariance functions for live weight, condition score, and dry-matter intake measured at different lactation stages of Holstein Friesian heifers. Livest. Prod. Sci. 57:67-77.

Korver, S. 1988. Genetic aspects of feed intake and feed efficiency in dairy cattle: A review. Livest. Prod. Sci. 20:1-13.

Lassen, J., N. A. Poulsen, M. K. Larsen, and A. J. Buitenhuis. 2016. Genetic and genomic relationship between methane production measured in breath and fatty acid content in milk samples from Danish Holsteins. Anim. Prod. Sci. 56:298-303.

Liinamo, A. E., P. Mantysaari, and E. A. Mantysaari. 2012. Short communication: Genetic parameters for feed intake, production, and extent of negative energy balance in Nordic Red dairy cattle. J. Dairy Sci. 95:6788-6794.

Manzanilla-Pech, C. I. V., R. F. Veerkamp, M. P. L. Calus, R. Zom, A. van Knegsel, J. E. Pryce, and Y. De Haas. 2014. Genetic parameters across lactation for feed intake, fat-and protein-corrected milk, and liveweight in first-parity Holstein cattle. J. Dairy Sci. 97:5851-5862.

Manzanilla-Pech, C. I. V., R. F. Veerkamp, R. J. Tempelman, M. L. van Pelt, K. A. Weigel, M. VandeHaar, T. J. Lawlor, D. M. Spurlock, L. E. Armentano, E. E. Connor, C. R. Staples, M. Hanigan, and Y. De Haas. 2016. Genetic parameters between feed-intakerelated traits and conformation in 2 separate dairy populations-the Netherlands and United States. J. Dairy Sci. 99:443-457.

Meuwissen, T. H. E.. B. J. Hayes, and M. E. Goddard. 2001. Prediction of total genetic value using genome-wide dense marker maps. Genetics 157:1819-1829.

Muller, C. J. C., S. W. P. Cloete, J. J. Olivier, J. A. Botha, and H. de Waal. 2006. Heritability of live weight and condition score in a Holstein herd and correlations with milk traits-Preliminary estimates. S. Afr. J. Anim. Sci. 36:79-88.

Pryce, J. E., W. J. Wales, Y. de Haas, R. F. Veerkamp, and B. J. Hayes. 2014. Genomic selection for feed efficiency in dairy cattle. Animal 8:1-10.

Pszczola, M., R. F. Veerkamp, Y. de Haas, E. Wall, T. Strabel, and M. P. L. Calus. 2013. Effect of predictor traits on accuracy of genomic breeding values for feed intake based on a limited cow reference population. Animal 7:1759-1768.

Robertson, A. 1959. The sampling variance of the genetic correlation coefficient. Biometrics 15:469-485.

Spurlock, D. M., J. C. M. Dekkers, R. Fernando, D. A. Koltes, and A. Wolc. 2012. Genetic parameters for energy balance, feed efficiency, and related traits in Holstein cattle. J. Dairy Sci. 95:5393-5402.

Su, G., P. Madsen, U. S. Nielsen, E. A. Mantysaari, G. P. Aamand, O. F. Christensen, and M. S. Lund. 2012. Genomic prediction for Nordic Red Cattle using one-step and selection index blending. J. Dairy Sci. 95:909-917.

ten Napel, J., M. P. L. Calus, M. Lidauer, I. Stranden, E. Mäntysaari, H. A. Mulder, and R. F. Veerkamp. 2016. MiXBLUP, user-friendly software for large genetic evaluation systems-Manual V2.02016-01. Wageningen, the Netherlands. Accessed Nov. 30, 2016. 
http://www.mixblup.eu/documents/Manual\%20MiXBLUP\%202 .0_January\%202016_V2.pdf.

Vallimont, J. E., C. D. Dechow, J. M. Daubert, M. W. Dekleva, J. W. Blum, C. M. Barlieb, W. Liu, G. A. Varga, A. J. Heinrichs, and C. R. Baumrucker. 2010. Genetic parameters of feed intake, production, body weight, body condition score, and selected type traits of Holstein cows in commercial tie-stall barns. J. Dairy Sci. 93:4892-4901.

Vallimont, J. E., C. D. Dechow, J. M. Daubert, M. W. Dekleva, J. W. Blum, C. M. Barlieb, W. Liu, G. A. Varga, A. J. Heinrichs, and C. R. Baumrucker. 2011. Heritability of gross feed efficiency and associations with yield, intake, residual intake, body weight, and body condition score in 11 commercial Pennsylvania tie stalls. J. Dairy Sci. 94:2108-2113.

Van Arendonk, J. A. M. 1991. Use of profit equations to determine relative economic value of dairy-cattle herd life and production from field data. J. Dairy Sci. 74:1101-1107.

Van Arendonk, J. A. M., G. J. Nieuwhof, H. Vos, and S. Korver. 1991 Genetic aspects of feed intake and efficiency in lactating dairy heifers. Livest. Prod. Sci. 29:263-275.

VanRaden, P. M. 2008. Efficient methods to compute genomic predictions. J. Dairy Sci. 91:4414-4423.

Veerkamp, R. F. 1998. Selection for economic efficiency of dairy cattle using information on live weight and feed intake: A review. J. Dairy Sci. 81:1109-1119.

Veerkamp, R. F., and S. Brotherstone. 1997. Genetic correlations between linear type traits, food intake, live weight and condition score in Holstein Friesian dairy cattle. Anim. Sci. 64:385-392.
Veerkamp, R. F., M. P. Coffey, D. P. Berry, Y. de Haas, E. Strandberg, H. Bovenhuis, M. P. L. Calus, and E. Wall. 2012. Genome-wide associations for feed utilisation complex in primiparous HolsteinFriesian dairy cows from experimental research herds in four European countries. Animal 6:1738-1749.

Veerkamp, R. F., J. K. Oldenbroek, H. J. Van Der Gaast, and J. H. J. Van Der Werf. 2000. Genetic correlation between days until start of luteal activity and milk yield, energy balance, and live weights. J. Dairy Sci. 83:577-583.

Veerkamp, R. F., and R. Thompson. 1999. A covariance function for feed intake, live weight, and milk yield estimated using a random regression model. J. Dairy Sci. 82:1565-1573.

Williams, Y. J., J. E. Pryce, C. Grainger, W. J. Wales, N. Linden, M. Porker, and B. J. Hayes. 2011. Variation in residual feed intake in Holstein-Friesian dairy heifers in southern Australia. J. Dairy Sci. 94:4715-4725.

Yang, J., B. Benyamin, B. P. McEvoy, S. Gordon, A. K. Henders, D. R. Nyholt, P. A. Madden, A. C. Heath, N. G. Martin, G. W. Montgomery, M. E. Goddard, and P. M. Visscher. 2010. Common SNPs explain a large proportion of the heritability for human height. Nat. Genet. 42:565-569.

Zom, R. L. G., G. Andre, and A. M. van Vuuren. 2012. Development of a model for the prediction of feed intake by dairy cows: 1 . Prediction of feed intake. Livest. Sci. 143:43-57. 